\title{
MATERIALES PARA UNA APROXIMACIÓN AL ESCOTISMO DE GILLES DELEUZE: UNIVOCIDAD DEL SER Y MODO INTRÍNSECO EN DIFERENCIA Y REPETICIÓN*
}

\author{
Héctor Hernando Salinas Leal * \\ doi: 10.11144/Javeriana.uph37-74.maed
}

\section{RESUMEN}

En este artículo se esboza la presencia del pensamiento de Juan Duns Escoto en Diferencia y repetición de Gilles Deleuze, teniendo como centro el análisis de los conceptos de "ser unívoco" y "modo intrínseco". La novedad del trabajo descansa en la preocupación por situar claramente el lugar de emergencia de la tesis sobre la univocidad del ser y la teoría del modo intrínseco que la acompaña en la obra de Duns Escoto, con el objetivo de determinar el horizonte de apropiación y el desplazamiento conceptual operados por Gilles Deleuze en su propia obra.

Palabras clave: Gilles Deleuze; Juan Duns Escoto; Diferencia y repetición; univocidad del ser; modo intrínseco

\footnotetext{
Me complace expresar mi agradecimiento a Gustavo Chirolla, por saber invitarme a participar en el Congreso Internacional 50 años de Différence et répétition de Gilles Deleuze (Bogotá, septiembre de 2018). Su intensidad vital sive filosófica hizo posible este texto.

" Pontificia Universidad Javeriana, Bogotá, Colombia.

Correo electrónico: hsalinas@javeriana.edu.co

Para citar este artículo: Salinas Leal, H. H. (2020). Materiales para una aproximación al escotismo de Gilles Deleuze: univocidad del ser y modo intrínseco en Diferencia y repetición. Universitas Philosophica, 37(74), 173-196. ISSN 0120-5323, ISSN en línea 2346-2426. doi: 10.11144/Javeriana. uph37-74.maed
} 


\title{
MATERIALS FOR APPROACHING GILLES DELEUZE'S SCOTISM: UNIVOCITY OF BEING AND INTRINSIC MODE IN DIFFERENCE AND REPETITION
}

\begin{abstract}
In this paper the influence of John Duns Scotus's thought in Gilles Deleuze's Difference and Repetition is outlined, centering the analysis in the concepts of "univocal being" and "intrinsic mode". The novelty of this work lies on its effort to accurately locate the thesis of the univocity of being and the theory of intrinsic mode that goes along with it in the work of Duns Scotus. Subsequently, an attempt is made to determine the horizon of appropriation and the conceptual displacement operated by Gilles Deleuze in his own work.
\end{abstract}

Keywords: Gilles Deleuze; Duns Scotus; Difference and Repetition; univocity of being; intrinsic mode 


\section{Introducción}

Si el título De eSte trabajo fuera "El espinozismo de Gilles Deleuze" o "El nietzscheanismo de Gilles Deleuze", es presumible que gran parte de su posible público lector tendría un punto de vista sólido y argumentado sobre el asunto. Sin embargo, la fórmula "el escotismo de Gilles Deleuze" resulta mucho más enigmática. Una respuesta sencilla y expedita al enigma que plantea la pregunta por el escotismo de Gilles Deleuze no es otra que presuponer que dicho escotismo es deleuziano. $\mathrm{Al}$ menos nos podemos atener a eso.

Sin embargo, esta constatación nos permite desde un comienzo detectar el lugar donde se plantea el problema que pretendemos poner de manifiesto. Nuestra contemporaneidad está mucho más familiarizada con Spinoza, Kant, Nietzsche, Bergson o el mismo Deleuze que con Juan Duns Escoto, el franciscano de finales el siglo XIII y comienzos del XIV que revolucionó la metafísica y explicitó el clamor del ser ya cantado por Parménides, en lo que algún historiador de la filosofía medieval llamó "el segundo comienzo de la metafísica" (Honnefelder, 2002, pp. 1-19).

Por esa razón, exponer el asunto relativo al escotismo de Deleuze consiste menos en preguntarnos por la filosofía de Deleuze, al menos en este trabajo, que por la de Duns Escoto en su interacción con la de Deleuze. En otros términos, intentamos aclarar los puntos centrales del pensamiento de Duns Escoto como materiales posibles para la obra de Deleuze y para su interpretación. Para nosotros, contemporáneos de nuestros contemporáneos, Duns Escoto es un nombre vacío, limitado tal vez a un par de nociones sin horizonte, como "univocidad del ser" o "haecceidad". Por ello, el objetivo de este trabajo es, en cierto modo, doble: intentar sacar a Duns Escoto del circuito de sus expertos, para leerlo de nuevo en clave de su futura apropiación deleuziana, y, al mismo tiempo, presentar y explicitar un conjunto de textos que, creemos, pueden facilitar la comprensión

1 Para una introducción al pensamiento de Duns Escoto son de provecho Williams, 2003, Ingham \& Dreyer, 2004, Sondag, 2005, y Merino, 2007. Mención aparte merece el trabajo clásico de Étienne Gilson, 1952, Jean Duns Scot: Introduction à ses positions fondamentales, pues, además de seguir constituyendo una razonable introducción al pensamiento escotista, fue también una de las fuentes de Deleuze en su comprensión del franciscano escocés. 
del proyecto deleuziano para quienes ya están familiarizados con él, pero no han tenido ocasión de recorrer su veta escotista.

Nuestro propósito temático será, pues, presentar las impresiones de un historiador de la filosofía medieval que se enfrenta a la lectura de algunas páginas célebres de Diferencia y repetición, en las que el pensamiento de Duns Escoto se articula a una historia de la metafísica narrada en tres tiempos. En dicha historia, la tesis sobre la univocidad del ser, y la teoría del modo intrínseco que la acompaña, se descubren y amplifican en un arco que va de Escoto a Nietzsche, pasando por Spinoza, para proclamar, en palabras de Deleuze, que "el ser unívoco es a la vez distribución nómade y anarquía coronada” (2002, p. 75).

Nuestra propuesta consiste en remontar las aguas torrenciales que arrastran esta afirmación para dirigirnos al texto clásico en el que Duns Escoto formula dicha tesis y poder preguntarnos de nuevo hoy ¿qué es entonces esa univocidad del ser?, ¿de dónde procede?, ¿qué preguntas la hicieron posible? Y, solo entonces, sobre ese suelo, quizá no muy sólido, preguntarnos cómo la lee Deleuze y desde dónde la articula con su proyecto. Así, estas preguntas, y sus respuestas, quizás permitan en otro momento -o a otros investigadores- volver a preguntar por el sentido de ciertos conceptos clave del proyecto deleuziano. Advertimos, sin embargo, que en el recorrido que aquí proponemos tendremos que renunciar, por razones de densidad y espacio, a tratar la teoría de la haecceidad en Duns Escoto, cuyo influjo en el pensamiento de Deleuze es innegable (véase, por ejemplo, Morejón, 2018).

Adicionalmente, una tesis no explícita y de mayor alcance recorrerá las observaciones que siguen: la noción de lo contemporáneo no cobija exclusivamente el presente y su pasado reciente. Sin ampliar el territorio de nuestra contemporaneidad filosófica, en el espíritu de la comprensión agambeniana de lo contemporáneo (Agamben, 2011, pp. 17-27), hasta incluir en él algunas configuraciones de la filosofía medieval (Salinas, 2015, pp. 345-367; Boulnois, 2016, pp. 321-350), por no mencionar la filosofía tardo-antigua en sus vertientes pagana y cristiana, será muy difícil construir o será deformada la idea que nos hagamos de lo que llamamos filosofía contemporánea y, específicamente, en este caso, de la filosofía de Gilles Deleuze, cuyo pensamiento, como todo pensamiento vigoroso y creador, no solo modifica el presente, sino que modifica también el futuro y el pasado de la filosofía. 


\section{2. "El ser es unívoco"}

EN LAS PRIMERAS PÁGINAS consagradas a "la diferencia en sí misma", primer capítulo de Diferencia y repetición, Deleuze describe los lugares constituyentes de la definición de la diferencia como diferencia conceptual, es decir, de la diferencia en tanto inscrita bajo el concepto en general. Advertía al final de la introducción a la obra, que "mientras se inscriba la diferencia en el concepto en general, no tendremos ninguna Idea singular de la diferencia, permaneceremos tan solo en el elemento de una diferencia ya mediatizada por la representación" (2002, p. 58).

Sobre ese territorio del concepto dividido por la diferencia y al que ella se somete, introduce Deleuze la tesis de la univocidad del ser que permitirá, precisamente, construir un concepto de la diferencia en sí misma, evocando, entre otros, el nombre de Duns Escoto. Recordemos el famosísimo pasaje en el que Deleuze introduce la tesis de la univocidad del ser en Diferencia y repetición.

Nunca hubo más que una proposición ontológica: el Ser es unívoco. No hubo nunca más que una ontología, la de Duns Escoto, que da al ser una sola voz. Decimos Duns Escoto porque supo llevar el ser unívoco al más alto grado de sutileza, corriendo el riesgo de dotarlo de abstracción. Pero de Parménides a Heidegger, se retoma la misma voz, en un eco que forma por sí solo todo el despliegue de lo unívoco. Una sola voz constituye el clamor del ser (Deleuze, 2002, p. 71).

Para Badiou (1997), en su Deleuze: el clamor del ser, "estamos aquí en el corazón del pensamiento deleuziano" (p. 41). Toda su filosofía giraría en torno a la ratificación de esta sentencia, "el ser es unívoco". Expresado en términos de proyección y larga duración, con respecto a esta tesis "lo esencial es que comporta consigo la afirmación de la inmanencia" (Zourabichvili, 2003, p. 82). Evidentemente, nos resulta muy claro que el énfasis tendrá que recaer sobre la sentencia misma y no sobre sus configuraciones concretas en Duns Escoto, Espinoza o Nietzsche, figuras sucesivas en una historia de la filosofía que despliega la virtualidad contenida en dicha univocidad.

$\mathrm{Y}$, sin embargo, a pesar de que Escoto no es más que aquel que "supo llevar el ser unívoco al más alto grado de sutileza”, con lo que Deleuze matizaría la afirmación según la cual nunca hubo más que la ontología de Escoto, páginas adelante 
dirá también, sin apenas parpadear, que el Opus Oxoniense es "el libro más grande de ontología pura" (2002, p. 77).

$\mathrm{Al}$ menos estas afirmaciones entusiastas invitan a darle una (h)ojeada al Opus Oxoniense, hoy más conocido como la Ordinatio, buscando eso que exaltó a Deleuze y que determinó su propio pensamiento: la constitución escotista de una ontología modal pura, por contraste y reacción a una de las formas de la analogía del ser, la expuesta por Enrique de Gante.

\section{El ser unívoco en el Opus Oxoniense de Duns Escoto}

La merecida Celebridad de Duns Escoto se expresa, en el terreno de la metafísica, por su postulación y defensa de la tesis de la univocidad del ser (Ordinatio I, d. 3, p. 1, qq. 1-3, ed. Vat. III, 1-123; véase Boulnois, 1988) y del modo intrínseco o distinción modal que la acompaña -la distinción formal y la haecceidad como principio de individuación-. Tras siglos de buscar solución a la problemática aristotélica del ser (Aubenque, 1962), enfatizando su equivocidad (Porfirio, Isagoge, II,10) o su analogía (por ejemplo en Tomás de Aquino, Summa theologiae, I, q. 13), Escoto sostendrá que Dios y las criaturas pueden ser conocidos bajo un concepto univoco a ambos (Ordinatio I, d. 3, p. 1, qq. 1-2, n. 26, ed. Vat. III, 18), modificando fundamentalmente esa tradición consolidada que consideraba la unidad de lo existente en términos de analogia entis (Courtine, 2005; Lonfat, 2004).

La univocidad del ser no se propone como respuesta a una pregunta o en una obra que nosotros consideraríamos consagrada a la ontología pura. La tesis de la univocidad del ser se presenta como única alternativa posible para resolver satisfactoriamente el problema de la cognoscibilidad de Dios para un intelecto finito dependiente del conocimiento sensible. Su presentación y defensa tiene lugar en la Opus Oxoniense u Ordinatio, que no es otra cosa que un comentario al libro de las Sentencias del teólogo del siglo XII Pedro Lombardo. Se trata, recordémoslo para situar el lugar de dicho texto, del manual de estudio de la teología más importante durante la Edad Media y vigente como texto de comentario al menos hasta el siglo XVI (Rosemann, 2004, pp. 194-211). Así, pues, ni la pregunta (que pertenece al campo de la teología natural), ni la obra 
(que se inscribe en la tradición del comentario medieval a las Sentencias) tenían como finalidad explícita fundar o defender una ontología pura.

En dicho comentario, Escoto se pregunta, como muchos otros lo han hecho antes que él, "si Dios es naturalmente cognoscible por el intelecto del viador" (Ordinatio I, d. 3, p. 1, qq. 1-2; ed. Vat. III, 1). El viador es quien está en tránsito, durante el lapso que cubre el nacimiento y la muerte, hacia la vida, feliz o desdichada, que lo espera después de la muerte. En otros términos, Escoto se pregunta por la posibilidad del conocimiento de Dios a partir de la situación del hombre y la capacidad natural de su intelecto en la vida presente.

La solución a esta pregunta defiende la posibilidad de un conocimiento natural y positivo de Dios, expresada en la constitución de su concepto en el intelecto del viador. Ahora bien, la pregunta acerca de "si Dios es naturalmente cognoscible por el intelecto del viador" está motivada por la tensión existente entre la capacidad natural del intelecto y el fin último de la metafísica como conocimiento de Dios y acceso a la felicidad. ¿Por qué hay tensión? Porque la capacidad natural del intelecto tiene por fundamento el conocimiento sensible y este fundamento parece impedir e incapacitar al intelecto para alcanzar un conocimiento de Dios y, por tanto, parece imposibilitar no solo el proyecto de la metafísica como ciencia sino, con ello, el acceso natural a la felicidad.

El drama que escenifica la debilidad del intelecto frente al objeto más digno de estima será desactivado por Escoto con la univocidad del ser.

\section{La definición de la univocidad}

LA POSICIÓN DE LA TESIS, antes de presentar los argumentos que la sostienen, está acompañada de una nota aclaratoria que explicita y delimita lógicamente su espacio de emergencia:

Digo que Dios no solo es concebido en un concepto análogo al concepto de la creatura, a saber, que sea absolutamente distinto a aquel que se predica de la creatura, sino en un concepto univoco a él y a la creatura. Y para que no haya controversia a propósito del nombre de univocidad, llamo concepto unívoco a aquel que es uno de tal modo que su unidad basta para la contradicción, al afirmar y negar lo mismo de lo mismo; y también basta, en tanto medio de un silogismo, para que se concluyan los extremos unidos en un [término] medio 
de tal modo uno, que [los extremos] se unen entre sí sin falacia de equivocación (Ordinatio I, d. 3, p. 1, qq. 1-2, n. 26, ed. Vat. III, 18; énfasis añadido).

Se imponen, al menos, cuatro observaciones a este pasaje. En primer lugar, la univocidad será la de un concepto, no la de un término con un único significado. Aquí nos confrontamos plenamente con una definición intencional de la univocidad que no tiene una correspondencia estricta con una univocidad semántica (la de una palabra o término que comporta uno y solo un significado). Así, lo unívoco no es el término ser en cuanto a su significado, sino el concepto mismo de ser en su dimensión intencional. Aunque esta noción parezca extraña, responde a la posición de otro autor, Enrique de Gante, que postulaba una analogía conceptual para el concepto de ser. Así, en esa misma dimensión intencional, la analogía que se critica no es la de un término con varios significados vinculados entre sí por un principio de atribución (como en Tomás de Aquino), sino una versión conceptual de la analogía, en la que, como veremos enseguida, dos conceptos distintos, pero muy cercanos, pueden dar lugar a una confusión del intelecto tal que este los asume como idénticos, como un único concepto, no siéndolo.

Así, en segundo lugar, la definición de la analogía implícita en la primera afirmación supone que Dios es concebido en un concepto de ser absolutamente distinto del concepto de ser en el que se concibe la creatura y que, sin embargo, es análogo a él. Una vez más, es determinante notar que en este pasaje no se alude a la definición de la analogía presente en la obra de Tomás de Aquino, en la que la analogía descansa en el descubrimiento de que los términos con los que nos referimos a Dios y a las criaturas ("Dios es bueno" y "Juan es bueno") comportan un significado (un concepto) que ni es totalmente idéntico (unívoco) ni absolutamente distinto (equívoco), conformándose sobre un terreno común establecido por la relación de causa a efecto entre Dios y sus criaturas, de tal modo que las perfecciones de Dios se manifiestan en las criaturas, en las que dichas perfecciones se diversifican (Summa theologiae, I, q. 13, a. 5).

De hecho, la alusión escotista apunta a la teoría de la analogía defendida por Enrique de Gante, interlocutor histórico de Duns Escoto, y sobre quien recaen, en distintos lugares de su obra, buena parte de sus críticas (Dumont, 1998; Pickavé, 2011; Porro, 2014). Sobre este autor habría que comenzar por señalar que su teoría de la analogía se construye como respuesta a una formulación de la univocidad, al menos implícita, contenida en la obra de Avicena. Veamos esto 
mejor. Para Enrique también se da una relación analógica entre Dios y las criaturas fundada en la causalidad; y los términos predicados de Dios y las criaturas no comportan ni un significado común (unívoco), ni un significado totalmente distinto (equívoco) a ellos. En este punto mantiene la posición de Tomás de Aquino. Sin embargo, Enrique enfrenta un adversario que Tomás de Aquino no tiene en cuenta. Para Avicena (1977), el primer concepto del intelecto es el concepto de ser, a partir de cuya determinación conocemos la diversidad de lo real, incluido Dios ${ }^{2}$. Esta tesis implícita, que será recuperada por Duns Escoto ${ }^{3}$, es inaceptable para Enrique de Gante, pues, en su opinión, quebraría la distancia ontológica entre Dios y la criatura, poniendo en peligro la trascendencia divina. Dicha consideración obliga a Enrique de Gante a acentuar la diversidad radical entre un concepto de ser que el hombre obtiene de las criaturas y otro que obtiene de Dios, de tal modo que un posible concepto común, unívoco, sería el resultado de la confusión producida en el intelecto por estos dos conceptos de ser ${ }^{4}$.

2 Avicena, Philosophia prima sive scientia divina, I, 5 (AvL, I, 31-33): "diremos entonces que cosa, ser (ens) y necesario son tales que inmediatamente se imprimen en el alma por una impresión primera, que no se adquiere a partir de otras [realidades] más conocidas que ellas [...]. En efecto, las [realidades] más aptas para ser concebidas por sí mismas son aquellas que son comunes a todas las cosas, como cosa y ser (ens) y uno, etc. Y, por lo tanto, de ningún modo puede manifestarse algo de ellas por una prueba que no sea circular, o por algo que sea más conocido que ellas. Es por ello que si alguien quisiera discurrir sobre ellas caerá en un embrollo, como aquel que dijo que la naturaleza del ser (certitudo entis) es o ser agente o ser paciente: aunque esta división sea del ser, sin embargo, el ser es más conocido que el agente y el paciente. En efecto, todos los hombres conciben la naturaleza del ser, pero ignoran si deba ser agente o paciente; y para mí, hasta ahora, esto no es manifiesto, sino solo por argumentación". Como lecturas introductorias a este punto en Avicena, véanse Bertolacci, 2006, Porro, 2006 y Lizzini, 2016.

3 En la sesión del 24 de marzo de 1981 de su curso sobre Spinoza, Deleuze (2017, pp. 489-494) establece este vínculo entre la teoría aviceniana de la indiferencia de la esencia y la teoría escotista de la univocidad del ser: "ven ustedes que Duns Escoto da contra Santo Tomás el mismo golpe al nivel del ser que le da Avicena al nivel de la esencia. A saber: el ser es unívoco. Aunque el ser se relacione a cosas que no tienen el mismo sentido -Dios y las criaturas-, no hay más que un único y mismo sentido de la palabra ser: ni finito ni infinito" (p. 492). Véase también An, 2013, pp. 60-71.

4 Enrique de Gante: "al tercer argumento, que el ser sin más (ens simpliciter) es concebido antes del concepto de ser que corresponde a Dios o a la creatura, hay que decir que no es verdad. En efecto, nunca puede concebirse algún concepto (intellectus) de ser sin más, sin que el hombre conciba algún concepto de Dios o de la creatura, a fin de que conciba algún concepto único simple y común a Dios y la creatura, además del concepto de Dios o de creatura, porque no puede concebirse tal 
En tercer lugar, el pasaje no defiende directamente la univocidad del ser, en su letra afirma que Dios es concebido en un concepto univoco a él y a la creatura. Esto significa que el concepto de Dios será cognoscible para el hombre en virtud de la determinación de dicho concepto unívoco aún no identificado. Desde ya podemos anticipar que, para Escoto, el concepto más perfecto y simple al que el hombre puede acceder para conocer a Dios es el concepto de ser infinito (Ordinatio I, d. 3, p. 1, qq. 1-2, n. 58; ed. Vat. III, 40; véanse también González Ginoccio, 2011; Cross, 2005, pp. 91-98; Catania, 1993).

Por último, la univocidad se define a partir de la unidad mínima necesaria exigida a un concepto para evitar la falacia de equivocación y para fungir como término medio de un silogismo, esto es, la unidad de su contenido. Así, la univocidad de un concepto se entiende desde su función lógica al servicio del discurso científico. Por ello, la univocidad del concepto de ser garantizará tanto la unidad de la ontología como la de la teología, pues la unidad del objeto garantiza la unidad de la ciencias.

concepto. Pero si el hombre concibe algo, este concepto o bien pertenece solamente al ser de Dios, o pertenece solamente al ser de la creatura. Pero el uno y el otro de estos [conceptos] es indiferente e igualmente simultáneo, desde la voz, para presentarse por naturaleza en su significado, que es ser. Por lo tanto, todo concepto real en el cual algo de una cosa se conciba concibiendo el ser sin más (ens simpliciter), o es el concepto de la cosa que es Dios (aut est conceptus rei quae Deus est) o de la cosa que es la creatura (aut quae creatura est), no de algo común al uno y al otro. Sin embargo, esto parece [ocurrir] a los que no pueden distinguir la multiplicidad del ser (multiplicitatem entis) y el ser del creador del ser de la creatura (et esse creatoris ab esse creaturae)" (Summae, art. 21, q. 2; I, 124v O).

5 Como lo afirma desde su obra temprana, las Cuestiones a la Isagoge, el objeto de una ciencia, su subiectum, cumple con unos requisitos tales que de su unidad se deriva la unidad de la ciencia: "hay que observar que el objeto de una ciencia (subiectum scientiae) debe cumplir con tres condiciones: que su 'quid est' [la esencia] y su 'quia est' [la existencia] sean conocidos, pues hay que presuponerlos en la demostración, según se afirma en el libro primero de los Segundos analíticos (1,71a11-16). Segunda condición: que por medio de su 'quid est' sean demostradas en dicha ciencia las propiedades de su objeto (passiones subiecti). Tercera condición: que todas las otras [realidades] determinadas en dicha ciencia se reduzcan (reducantur) a su objeto y se consideren a causa de dicho objeto. De lo contrario, no habría unidad en dicha ciencia a partir de la unidad de su objeto (ab eius unitate non esset unitas scientiae)" (Escoto, In Porph., q. 3, n. 13; OPh, I, 14; énfasis añadido). 


\section{La neutralidad del ser unívoco}

SOBRe ESTA ACLARACIÓn y la definición propuesta de la univocidad, Escoto procede a formular su primer argumento de un concepto unívoco a Dios y a las criaturas, único argumento del que nos ocuparemos en este trabajo, pues su formulación despliega explícitamente la tesis de univocidad del ser:

Y pruebo la univocidad así entendida con cinco argumentos. Primero, así: todo intelecto que tiene certeza (certus) de un concepto y duda sobre otros, tiene un concepto sobre el cual tiene certeza que es distinto de los conceptos sobre los cuales tiene duda; el sujeto incluye al predicado. Pero el intelecto del viador puede tener certeza de que Dios es un ser (ens), dudando de que sea finito o infinito, creado o increado; por lo tanto, el concepto de ser (ens) respecto de Dios es distinto al concepto de este y aquel [finito o infinito, creado o increado], y así, es neutro por síy se incluye en el uno y en el otro; por lo tanto, es unívoco (Ordinatio I, d. 3, p. 1, qq. 1-2, nn. 27-29; ed. Vat. III, 18-19).

El argumento distingue, en primer lugar, un concepto concebido con absoluta certeza de otro concepto concebido con duda o cuya certeza es dudosa. Esta premisa es evidente por sí misma, o en los términos de Escoto, el sujeto incluye al predicado. En seguida, presenta un caso en el que se presenta esta tajante distinción: Dios concebido como ser, de un lado, y la determinación de ese ser como finito o infinito, creado o increado, del otro. Dada la distinción, dicho concepto de ser es anterior e indiferente a estas determinaciones y por tanto es unívoco. En otras palabras, teniendo certeza de que Dios es y dudando de si es finito o infinito, creado o increado, descubrimos que dicho concepto de ser, bajo el cual concebimos a Dios, no incluye necesariamente sus determinaciones y que, por el contrario, es anterior, neutro e indeterminado con respecto a la finitud o infinitud. Por ello, el concepto es unívoco y, en su comunidad, recubre la totalidad de lo real, pues las determinaciones finito o infinito, creado o increado, se entienden como trascendentales disyuntivos. El ser unívoco es convertible con la disyunción "ser finito o ser infinito".

Así, pues, los efectos filosóficos de dicha tesis son incalculables. Menciono rápidamente los tres más importantes. Se le da un sólido fundamento, por fin, a la posibilidad de la metafísica como ciencia, específicamente, en nuestros términos, 
como ontología ${ }^{6}$ (y quizá en ese sentido puede leerse, como lo hace Deleuze, la tesis de la univocidad como una figura eminente de la ontología pura). Las indecisiones del texto aristotélico, es conocido, conducen a la imposibilidad de identificar el objeto de la metafísica y con ello a la imposibilidad de su fundación como ciencia. Con la univocidad del ser, quizá por primera vez, se abre el horizonte a la metafísica como ciencia del ser en tanto ser, entendiendo por ello no un término con múltiples significaciones, sino un concepto con el mínimo necesario de unidad para garantizar la posibilidad del discurso científico.

La segunda consecuencia se sitúa en el terreno de la teología natural. Desde ahora, la ciencia de Dios también se funda sobre el suelo sólido de la determinación del ser de Dios, como ser infinito. Lo que emerge con la tesis de Duns Escoto es una nueva figura de la metafísica como ontología apuntalada sobre la unidad de su objeto, el ser en tanto ser, y dirigida a su finalidad, el conocimiento de Dios en tanto ser infinito. Finalmente, tal metafísica será una metafísica del concepto de ser y de lo que lo acompaña, menos inclinada a describir las relaciones causales reales entre los seres que sus relaciones esenciales o conceptuales.

Con Escoto, se alcanza uno de los lugares más sofisticados de lo que Étienne Gilson (2001) llama "la metafísica de la esencia” (pp. 107-146), esto es, una metafísica concentrada en el estudio de los rasgos esenciales de lo real, en la que se neutraliza el punto de vista existencial y causal de su producción y de su principio. Y es por ello que el límite de dicha ontología, desde el punto de vista de Deleuze, es esa neutralidad, que lo sustrae de una "afirmación pura" de la vida y la existencia (tarea que Spinoza acometerá en el siguiente momento de esta historia). En palabras de Deleuze:

Pero el ser unívoco está pensado como neutro, neuter, indiferente a lo infinito y a lo finito, a lo singular y a lo universal, a lo creado y a lo increado. Escoto merece, pues, el nombre de "doctor sutil", porque su mirada discierne el ser más acá del entrecruzamiento de lo universal y de lo singular. Para neutralizar las fuerzas de analogía en el juicio, se adelanta y neutraliza en primer lugar el ser en un concepto abstracto. Tal es el motivo por el cual se ha limitado a

6 Para una comprensión del punto de partida de la especulación escotista sobre el objeto de la metafísica, véase Salinas, 2016. 
pensar el ser unívoco. Vemos cuál es el enemigo del cual trata desesperadamente de escapar, de acuerdo con las exigencias del cristianismo: el panteísmo, en el cual caería si el ser común no fuese neutro (2002, p. 77).7

\section{Trascendencia o inmanencia: el papel del modo intrínseco}

En eSTE PUnto, Deleuze contesta un conjunto de preguntas hasta ahora no explícitas: ¿por qué tomó tantos siglos postular la univocidad del ser?, ¿ por qué prevaleció durante tantos siglos, con sus variantes, la analogía?, ¿qué impedía obstinadamente dar el paso de la una a la otra? La analogía permitía establecer un vínculo entre Dios y las criaturas a partir de la causalidad creadora de Dios y, simultáneamente, permitía mantener la distancia ontológica entre Dios y las criaturas, expresada en el concepto de trascendencia divina. Con la univocidad, nos hallamos en un territorio totalmente nuevo, pues Dios y las criaturas se incluyen bajo un mismo concepto y se delimitan por una determinación intrínseca del mismo en la pareja disyuntiva ser infinito/ser finito. Ahora bien, en este nuevo escenario, ¿cómo se salvaguardaría la trascendencia divina, si el ser, en su comunidad y unidad, es anterior, neutro e indiferente a sus determinaciones?

7 De aquí que el segundo momento de esta historia consista en la desactivación de la neutralidad del ser univoco o en la activación de su afirmación pura: "con el segundo momento, Spinoza opera un progreso considerable. En lugar de pensar el ser unívoco como neutro o indiferente, hace de él un objeto de afirmación pura. El ser unívoco se confunde con la sustancia única, universal e infinita: está enunciado como Deus sive Natura" (2002, p. 78). Como en Diferencia y repetición, y publicado también en 1968, en Spinoza y el problema de la expresión, Deleuze da cuenta de la "grande tradition" (la historia) en la que se inscribe el método y el proyecto general de Spinoza, en un gesto que resuena con lo ocurrido en las páginas de Diferencia y repetición que aquí comentamos. En la obra sobre Spinoza (Deleuze, 1996) se lee lo siguiente: "El método de Spinoza no es ni abstracto ni analógico. Es un método formal y de comunidad. Opera por nociones comunes; luego toda la teoría spinozista de las nociones comunes halla su principio precisamente en ese estatuto del atributo. Si finalmente hay que dar nombre a ese método, como a la teoría subyacente, se reconocerá fácilmente en él la gran tradición de la univocidad. Creemos que la filosofía de Spinoza permanece en parte ininteligible si no se ve en ella una lucha constante contra las tres nociones de equivocidad, eminencia y analogía. [...] En nada creemos menguar la originalidad de Spinoza reemplazándole en una perspectiva que ya era la de Duns Scoto. Debemos dejar para más adelante el análisis de cómo Spinoza interpreta por su cuenta la noción de univocidad, y cómo la comprende de una manera totalmente diferente a la de Duns Scoto" (pp. 42-43). 
O, formulado de otro modo, ¿̨por qué no hay panteísmo? ¿Por qué no se disuelve la naturaleza divina con la creada en un único ser?

Para responder esta pregunta, volvamos a Deleuze, cuando despliega uno de los efectos centrales de la univocidad del ser y de sus determinaciones:

Lo esencial de la univocidad no es que el Ser se diga en un único y mismo sentido, sino que se diga, en un único y mismo sentido, de todas sus diferencias individuantes o modalidades intrínsecas. El ser es el mismo para todas esas modalidades, pero esas modalidades no son las mismas. Es "igual" para todas, pero ellas mismas no son iguales. Se dice en un solo sentido de todas, pero ellas mismas no tienen el mismo sentido. Es propio de la esencia del ser unívoco referirse a diferencias individuantes, pero estas diferencias no tienen la misma esencia y no varían la esencia del ser, así como el blanco se refiere a intensidades diversas, pero sigue siendo esencialmente el mismo blanco (Deleuze, 2002, p. 72)8.

En este pasaje, Deleuze ha introducido una noción, "modalidades intrínsecas”, que permite responder a la pregunta formulada un poco antes. ¿Cómo se salvaguarda la trascendencia divina en un modelo en el que el ser se dice unívocamente de todo aquello que lo contiene? ¿Cómo será Dios distinto a la criatura si en ambos casos y en el mismo sentido los llamamos "ser"?

En un lenguaje que es el suyo, no se equivoca Deleuze en su interpretación de Escoto, cuando apunta a esa aparente contradicción que permite convivir a la univocidad del ser con la multiplicidad de los seres. El ser, en su comunidad, neutralidad, simplicidad e indeterminación, está incluido en el concepto de todo ser determinado, como el ser finito y el ser infinito. Pero ¿por qué razón podemos afirmar que se trata siempre del mismo ser y no de un ser contraído por una determinación o diferencia extrínseca, que daría lugar a una multiplicidad

8 En Spinoza y el problema de la expresión (Deleuze, 1996), encontramos también esta reflexión, en función de la lectura de Spinoza: "Duns Scoto es sin duda quien más lejos llevó la empresa de una teología positiva. Denuncia a la vez la eminencia negativa de los neo-platónicos, la pseudoafirmación de los tomistas. Les opone la univocidad del ser: el ser se dice en el mismo sentido de todo lo que es, infinito o finito, aunque no sea bajo la misma 'modalidad'. Pero precisamente, el ser no cambia de naturaleza al cambiar de modalidad, es decir, cuando su concepto es predicado del ser infinito y de los seres finitos" (p. 56). 
no común o a un ser compuesto? Aquí tenemos que dirigir nuestra atención al concepto escotista de modo intrínseco.

El resultado más refinado de la teología natural escotista ha sido mostrar cómo el intelecto humano puede efectivamente tener un concepto positivo de Dios que no es otro que el concepto de ser infinito. Con este hallazgo, no solo Escoto resuelve la pregunta que suscitó la postulación de la univocidad del ser, sino que sus lectores entendemos cuál fue la motivación profunda (teológica) que dirigió la constitución de la metafísica como ontología pura ("si Dios es naturalmente cognoscible por el intelecto del viador"). Veamos:

Digo que podemos alcanzar numerosos conceptos propios de Dios, que no convienen a las creaturas; de tal suerte son los conceptos de todas las perfecciones absolutas tomadas en grado sumo. Y el concepto a la vez másperfecto $y$ más simple que nos es posible es el concepto de ser infinito.

En efecto, este es más simple que el concepto de ser bueno, ser verdadero u otros semejantes, porque "infinito" no es un cuasi atributo o una pasión del ser o de aquello de lo que él es predicado, sino que da cuenta del modo intrínseco de esta entidad, de tal modo que, cuando digo "ser infinito", no poseo un concepto como por accidente, a partir de un sujeto y una pasión, sino un concepto por sí del sujeto en un cierto grado de perfección, es decir, de infinitud. Del mismo modo, una blancura intensa no indica un concepto por accidente como "blancura visible"; al contrario, la intensidad da cuenta de un grado intrínseco de blancura en sí. Y así la simplicidad de este concepto de ser infinito es manifiesta (Escoto, Ordinatio I, d. 3, p. 1, qq. 1-2, n. 58; ed. Vat. III, 40; énfasis añadidos).

Como podemos constatar, en este pasaje vemos cómo se articulan el concepto de ser con su modo intrínseco a través del concepto de intensidad. Nos suena familiar, ¿no es verdad? Tenemos aquí, operando, una trilogía de conceptos que será central en el proyecto de Deleuze. Vemos cómo el ser es el mismo y la blancura es la misma, aunque estén determinados intrínsecamente por un cierto grado de intensidad (o cantidad intensiva) que no se agrega al ser o la blancura desde fuera, sino que expresa, como diría Deleuze, su potencia intrínseca. De este modo, el concepto mismo no pierde su simplicidad como en la composición de un sujeto y su atributo. Al contrario, en todos los casos se trata del mismo 
ser o de la misma blancura, de la misma esencia, más o menos intensificada por sus modos intrínsecos, en un modelo en el que se conserva la univocidad dando lugar simultáneamente a la multiplicidad, sin contradicción aparente.

\section{Ser, atributos y modos}

Ahora bien, todo el esfuerzo escotista va en la dirección de mostrar que no hay concepto de ser sin modo intrínseco, pero también que es posible considerar intelectualmente al concepto de ser independientemente de sus modos. Por ello, puede ser el mismo para todas sus modalidades, siendo sus modalidades distintas entre sí.

Cuando se entiende una cierta realidad con su modo intrinseco, ese concepto no es a tal punto absolutamente simple que no pueda ser concebido sin su modo, pero en ese caso se trata de un concepto imperfecto de esta cosa; también puede ser concebido bajo el modo y en ese caso es un concepto perfecto de la cosa. Ejemplo: si la blancura se hallara en el décimo grado de intensidad, por simple que ella fuera bajo todos sus modos en la cosa, ella podría sin embargo ser concebida bajo la razón de tanta blancura y en ese caso sería concebida perfectamente, por un concepto adecuado a la cosa misma. O bien, ella podría ser concebida exclusivamente bajo la razón de blancura, y en ese caso sería concebida por un concepto imperfecto y al que le faltaría la perfección de la cosa; y tal concepto imperfecto podría ser común a esta blancura y a otra, mientras que un concepto perfecto le seria propio.

Se requiere entonces una distinción, entre aquello de donde se recibe el concepto común y aquello de donde se recibe el concepto propio, no como una distinción de realidad a realidad, sino como la distinción entre una realidad y sus modos propios e intrínsecos; y esta distinción es suficiente para tener un concepto perfecto o imperfecto de la misma cosa, porque lo imperfecto es común y lo perfecto es propio. Pero los conceptos de género y de diferencia requieren una distinción entre [varias] realidades y no solamente entre la misma realidad concebida perfectamente e imperfectamente (Escoto, Ordinatio I, d. 8, p. 1, q. 3, nn. 138-139; ed. Vat. IV, 222-223; énfasis añadidos). 
El concepto unívoco de ser concebido sin sus modos es, pues, imperfecto y común; determinable por ellos. Pero lo más destacable de este pasaje es la tematización del tipo de distinción requerida entre el concepto común y sus modos, pues no es ni una distinción real (de realidad a realidad), ni una distinción de razón (producida exclusivamente por el intelecto), es una distinción modal. Con ella, resulta posible distinguir grados de ser (sus intensidades) que no son, sin embargo, realidades independientes de dicho ser. De este modo, el concepto de ser infinito no resulta de la composición de dos realidades, sino de la consideración de una realidad en su grado sumo de intensidad.

En el Tratado sobre el Primer Principio (IV, 88, ed. Kluxen, pp. 116-118), Escoto no dudará en calificar dicha infinitud del ser como infinitud intensiva, por oposición a la infinitud extensiva. En otros términos, dicha infinitud no caería bajo la categoría de la cantidad y por tanto bajo el estudio de la física. La infinitud intensiva solo puede ser objeto de estudio de la metafísica como ontología y, en último término, de la teología natural. En palabras de Deleuze:

La distinción modal se establece entre el ser o los atributos, por una parte, y, por otra, las variaciones intensivas de las que son capaces. Estas variaciones, como los grados de lo blanco, son modalidades individuantes cuyo infinito y finito constituyen precisamente las intensidades singulares (2002, p. 77).

Ahora bien, esto no significa que el concepto de ser no tenga atributos propios, los tradicionalmente llamados atributos trascendentales. En un pasaje ya citado se alude al ser como bueno, o como verdadero. En este caso, los atributos del ser, que tampoco son distinguibles de él como se distingue una realidad de otra, comportan otro tipo de distinción: la distinción formal. Aunque inseparables en la realidad, los atributos del ser dan cuenta de aspectos de dicha realidad (de formalidades suyas) que pueden ser tratadas independientemente unas de otras, sin comprometer la unidad de su sujeto. Al respecto, la explicación de Deleuze es impecable:

La distinción formal es, en efecto, una distinción real, puesto que se funda en el ser o en la cosa, pero no es necesariamente una distinción numérica, porque se establece entre esencias o sentidos, entre "razones formales" que pueden dejar subsistir la unidad del sujeto al cual se las atribuye. Así, no solo la univocidad del ser (con relación a Dios y a las criaturas) se prolonga en 
una univocidad de los "atributos", sino que, bajo la condición de su infinitud, Dios puede poseer esos atributos unívocos formalmente distintos, sin perder nada de su unidad (1968a, pp. 57-58; 2002, p. 77).?

Citemos un último pasaje de la Ordinatio, en el que Escoto explica por qué no puede identificarse al ser unívoco y sus modos intrínsecos con la pareja género/diferencia específica. En este pasaje nos encontraremos con el lugar en el que Deleuze vería la emergencia de una posible diferencia intrínseca, una diferencia no sometida al concepto en general.

El concepto de una especie no es solamente el concepto de una realidad y de los modos intrínsecos de esa realidad, porque en ese caso la blancura podría ser un género y los grados intrínsecos de la blancura podrían ser diferencias específicas. Los [modos] por los cuales algo común es contraído a Dios y a la criatura son lo finito y lo infinito, que dan cuenta de los grados intrínsecos de aquellos. Por lo tanto, tales contractantes no pueden ser diferencias y no constituyen con lo contraído un concepto compuesto como el concepto de especie debe ser compuesto. Al contrario, el concepto formado por el contraído y el contractante es más simple de lo que puede serlo el concepto de especie (Escoto, Ordinatio I, d. 8, p. 1, q. 3, n. 108; ed. Vat. IV, 202-203).

Ser infinito no es una expresión que esconda la pareja género/diferencia específica (como animal racional); expresa, por el contrario, la pareja entidad/modo intrínseco. En la medida en que los modos son intrínsecos a la realidad que modifican, no pueden identificarse con diferencias específicas, ni dichas realidades con géneros. La relación es más simple, dice Escoto. Esto implica que aún nos enfrentamos a una entidad numéricamente una y a sus grados de intensidad; y no a dos entidades que se componen para formar una tercera más compleja que las dos primeras.

\section{La historia de la filosofía}

"LA HISTORIA DE LA FILOSOfía determina tres momentos principales en la elaboración de la univocidad del ser" nos dice Deleuze (2002, p. 77). Estos tres

9 Véase el tratamiento paralelo de la distinción formal en Spinoza y el problema de la expresión (Deleuze, 1996, pp. 57-60). 
momentos tienen nombre propio: Escoto, Spinoza, Nietzsche. Una historia en la que cada uno construye sobre lo construido, modificando simultáneamente los cimientos de la obra en marcha. Sin embargo, en esta historia falta un nombre, el de su historiador, Gilles Deleuze.

Por esta razón, teniendo en cuenta estos pocos indicios, me gustaría preguntarme brevemente por el estatuto de la historia de la filosofía en Diferencia y repetición. Someto a consideración del lector tres dimensiones en las que Deleuze se relaciona con la historia de la filosofía.

En primer lugar, está la historia de la filosofía, entendida como la práctica profesional y doctrinal, en la que él mismo fue educado. La historia de la filosofía como "agente de poder", como "represora" del pensamiento. Una imagen del pensamiento "que impide que las personas piensen" (Deleuze \& Parnet, 1980, p. 17; véase también Deleuze, 1992, p. 13).

En segundo lugar, está la historia de la filosofía que él mismo practica en sus monografías. "Prefería a ciertos autores que daban la impresión de formar parte de la historia de la filosofía, pero que en realidad escapaban a ella en ciertos aspectos o en todos: Lucrecio, Spinoza, Hume" (Deleuze \& Parnet, 1980, p. 19). Su estrategia, por lo demás, consistía en hacerle decir al autor algo que no ha dicho, pero que contiene virtualmente.

Me imaginaba acercándome a un autor por la espalda y dejándole embarazado de una criatura que, siendo suya, sería sin embargo monstruosa. Era muy importante que el hijo fuera suyo, pues era preciso que el autor dijese efectivamente todo aquello que yo le hacía decir; pero era igualmente necesario que se tratase de una criatura monstruosa, pues había que pasar por toda clase de descentramientos, deslizamientos, quebrantamientos y emisiones secretas, que me causaron gran placer (Deleuze, 1992, p. 14).

Y, por último, están sus observaciones y su práctica de la historia de la filosofía en Diferencia y repetición, que pertenecerían al segundo caso, en el que hace decir a los autores un impensado latente en ellos, pero que se modifica al hacerlos girar en torno a un problema en el que convergen.

En ese sentido, para el caso de la historia de la determinación de la univocidad del ser, los tres momentos (Escoto, Spinoza, Nietzsche) constituyen una forma de progresión hacia la concepción deleuziana del ser unívoco. 
Sin embargo, dicha progresión debería leerse de un modo más sutil, indicado por el mismo Deleuze en el prefacio de Diferencia y repetición, a partir de la exigencia impuesta por los nuevos medios de expresión filosófica inaugurados por Nietzsche:

En ese sentido, podemos desde ahora plantear el interrogante de la utilización de la historia de la filosofía. Nos parece que la historia de la filosofía debe desempeñar un papel bastante análogo al de un collage en la pintura. La historia de la filosofía es la reproducción de la filosofía misma. Sería necesario que la exposición, en historia de la filosofía, actúe como un verdadero doble y contenga la modificación máxima propia del doble (imaginamos un Hegel filosóficamente barbudo, un Marx filosóficamente lampiño, con las mismas razones que una Gioconda bigotuda) [...]. Las exposiciones de historia de la filosofía deben representar una suerte de cámara lenta, de cristalización o de inmovilización del texto: no solo del texto al cual se refieren, sino también del texto en el cual se insertan. De este modo, tienen una existencia doble y, como doble ideal, la pura repetición del texto antiguo y del texto actual el uno dentro del otro. Tal es el motivo por el cual hemos tenido, a veces, que integrar las notas históricas en nuestro texto mismo, para poder, así, acercarnos a esta doble existencia (2002, p. 18).

No nos resulta difícil imaginar un Escoto nómade y anárquico como doble de ese otro del que he venido hablando, justamente en la medida en que las piezas del collage se leen en su interacción espacial, por el contraste que generan unas con otras y por la figura que genera el conjunto. De cualquier manera, desde su propio punto de vista, en tanto sustancia concreta, él mismo, Duns Escoto, estaba sometido a la relación sustancia, atributos y modos intrínsecos.

Sin embargo, un historiador de la filosofía convencional, como el autor de este trabajo, recordará al final de este recorrido que en Duns Escoto el concepto unívoco de ser aún es el concepto que puede constituir un intelecto; que en Duns Escoto aún hay un yo y aún hay Dios; que aún hay naturaleza común y universal lógico (Salinas, 2019); que aún hay haecceidad, entendida como última actualidad formal de esta sustancia concreta; y, quizá lo central aquí, que la metafísica, como ontología, aún no está en movimiento. Por el contrario, la ontología fija las condiciones del movimiento, pero no es ella misma móvil. En Duns Escoto, el movimiento aún pertenece al campo de la física. 


\section{Conclusiones}

CON LO QUE AQUí HAYAMOS PODIDO GANAR en un acercamiento al escotismo de Gilles Deleuze, aún resulta imprescindible explicar el progreso que supone abandonar la analogía por la univocidad -cómo la univocidad abre la vía para la liberación de la diferencia del concepto en general y cómo la analogía es condición de su sometimiento a dicho concepto-. También es preciso explicar el lugar que tendrá el principio de individuación escotista (la haecceidad) para la teoría deleuziana de la individuación (Leclercq, 2003). Esta tarea impone la lectura de Porfirio, Aristóteles y Tomás de Aquino, entre otros, en clave deleuziana. Nuestra intención no ha sido otra que acercar los textos de Duns Escoto a su lugar de inserción en Diferencia y repetición, sabiendo que el alcance y la profundidad de dicha inserción queda aún por determinar y evaluar.

Por lo demás, el escotismo de Deleuze desborda ampliamente un análisis minucioso de lo ocurrido en Diferencia y repetición. Hay pistas, huellas o marcas profundas de esa presencia en Spinoza y el problema de la expresión (1996); en Lógica del sentido (1969, pp. 208-211) ${ }^{10}$; en Mil mesetas (Deleuze \& Guattari, 2008, pp. 257 258); o en el Curso sobre Spinoza ofrecido en Vincennes durante el año académico 1980-1981 (2017). Esta no pretende ser una enumeración exhaustiva; simplemente indica otras líneas de exploración de dicha relación. Pero, en todos los casos, es mi opinión, la exploración debe seguir el modelo aquí utilizado, en el que el análisis de Deleuze se hace por contraste directo con los textos de Duns Escoto, pues es su resonancia conjunta la que nos permitiría ver su cercanía y su lejanía.

Referencias

Agamben, G. (2011). Desnudez. Barcelona: Anagrama.

An, J. (2013). Sur la transformation spinoziste de l'idée de transcendantal dans la première philosophie de Gilles Deleuze (Tesis doctoral inédita). Université de Toulouse II Le Mirail, Toulouse, Francia. (tel-00909529).

Aubenque, P. (1962). Le problème de l'être chez Aristote : Essai sur la problématique aristotélicienne. París: PUF.

10 Sobre la univocidad del ser en Lógica del sentido, véase el lúcido trabajo de Hernández, 2004. 
Avicena (1977). Liber de philosophia prima sive Scientia divina (Vols. I-IV). S. Van Riet (Ed.). Lovaina/Leiden: Peeters-Brill.

Badiou, A. (1997). Deleuze: El clamor del ser. Buenos Aires: Manantial.

Bertolacci, A. (2006). The Reception of Aristotle's Metaphysics in Avicenna's Kitāb al-Šifā: A Milestone of Western Metaphysical Thought. Leiden/Boston: Brill.

Boulnois, O. (1988). La destruction de l'analogie et l'instauration de la métaphysique. En: J. D. Scot. Sur la connaissance de Dieu et l'univocité de l'étant (pp. 11- 81). París: PUF.

Boulnois, O. (2016). ¿Qué hay de nuevo? La Edad Media. Universitas Philosophica, 33(67), 321-350. Dor: 10.11144/Javeriana.uph33-67.qnem

Catania, F. J. (1993). John Duns Scotus on ens infinitum. American Catholic Philosophical 2uarterly, 67(1), 37-54. DOI: 10.5840/acpq199367143

Courtine, J-F. (2005). Inventio Analogiae: Métaphysique et Ontothéologie. París: Vrin. Cross, R. (2005). Duns Scotus on God. New York: Routledge.

Deleuze, G. (1968). Différence et répétition. París: PUF.

Deleuze, G. (1969). Logique du sens. París: Minuit.

Deleuze, G. (1992). Conversaciones 1972-1990. (Trad. J. L. Pardo). Valencia: Pre-Textos.

Deleuze, G. (1996). Spinoza y el problema de la expresión. (Trad. H. Vogel). Barcelona: Muchkin

Deleuze, G. (2002). Diferencia y repetición. (Trad. M. S. Delpy \& H. Beccacece). Buenos Aires: Amorrortu.

Deleuze, G. (2017). En medio de Spinoza. Buenos Aires: Cactus.

Deleuze, G., \& Guattari, F. (2008). Mil mesetas. (Trad. J. Vásquez Pérez). Madrid: Pre-textos.

Deleuze, G., \& Parnet, C. (1996). Dialogues. París: Flammarion.

Deleuze, G., \& Parnet, C. (1980). Diálogos (Trad. J. Vásquez). Valencia: PreTextos.

Dumont, S. (1998). Henry of Ghent and Duns Scotus. En: J. Marenbon (Ed.), Routledge History of Philosophy. (Vol. III). Medieval Philosophy (pp. 131328). London: Routledge.

Enrique de Gante (1953). Summae quaestionum ordinariarum (Vols. I-II). E. M. Buytaert (Ed.). (Reimpresión de la edición de 1520). Nueva York/Lovaina/ Padenborn: The Franciscan Institute/E. Nauwelaerts/F. Shöningh. 
Gilson, É. (1952). Jean Duns Scot : Introduction à ses positions fondamentales. París: Vrin.

Gilson, É. (2001). El ser y los filósofos. Pamplona: Eunsa.

González Ginoccio, D. (2011). Ser e infinito en Duns Escoto. Pamplona: Eunsa. Hernández, J. P. (2004). La univocidad del ser: lenguaje y ontología en Gilles Deleuze. Universitas Philosophica, 21(43), 123-144.

Honnefelder, L. (2002). La métaphysique comme science transcendantale : entre le Moyen Age et les Temps modernes. (Trad. I. Mandrella, revisada por O. Boulnois, J. Greisch, \& Ph. Capelle). Paris: PUF.

Ingham, Mary Beth y Dreyer, Mechthild (2004). The Philosophical Vision of John Duns Scotus: An Introduction. Washington: The Catholic University of America Press.

Juan Duns Escoto (1954). Opera omnia (Vol. III). Ordinatio I, d. 3. C. Balić et al. (Ed.). Civitas Vaticana: Typis Polyglottis.

Juan Duns Escoto (1956). Opera omnia (Vol. IV). Ordinatio I, dd. 4-10. Balić et al. (Ed.). Civitas Vaticana: Typis Polyglottis.

Juan Duns Escoto (1999). Opera philosophica (Vol. I). Quaestiones in librum Porphyrii Isagoge et quaestiones super praedicamenta Aristotelis. R. Andrews et al. (Ed.). Saint Bonaventure (NY): The Franciscan Institute.

Juan Duns Escoto (2009). Tractatus de primo principio. W. Kluxen (Ed.). Darmstadt: Wissenschaftliche Buchgesellschaft.

Leclercq, S. (2003). La présence de Jean Duns Scot dans l'œuvre de Gilles Deleuze, ou la généalogie du concept d'heccéité. Symposium: Canadian Journal of Continental Philosophy/Revue canadienne dephilosophie continentale, 7(2), 143-158. DoI: $10.5840 /$ symposium 20037212

Lizzini, O. (2016). Ibn Sina’s Metaphysics. En: E. N. Zalta (Ed.), The Stanford Encyclopedia of Philosophy (ed. de otoño del 2016). Recuperado de https:// plato.stanford.edu/archives/sum2019/entries/ibn-sina-metaphysics/

Lonfat, J. (2004). Archéologie de la notion d'analogie d'Aristote à Saint Thomas d'Aquin. Archives d'histoire doctrinale et littéraire du Moyen Âge, 71, 35-107. DOI: $10.3917 /$ ahdlm.071.0035

Merino, J. A. (2007). Juan Duns Escoto: Introducción a su pensamiento filosóficoteológico. Madrid: BAC. 
Morejón, G. (2018). Differentiation and Distinction: On the Problem of Individuation form Scotus to Deleuze. Deleuze and Guattari Studies, 12(3), 353-373. DoI: $10.3366 /$ dlgs.2018.0316.

Pedro Lombardo. (1971-1981). Sententiae in IV libris distinctae. 2 Vols. Ignatius Brady, O. F. M. (Ed.). Grottaferrata: Editiones Collegii S. Bonaventurae Ad Claras Aquas.

Pickavé, M. (2011). Henry of Ghent on Metaphysics. En: G. A. Wilson (Ed.), $A$ Companion to Henry of Ghent (pp. 153-179). Leiden/Boston: Brill.

Porfirio (1998). Isagoge. (Texto griego, texto latino y traducción francesa deA. de Libera y A.-Ph. Segonds; Introducción de A. de Libera). París: Vrin.

Porro, P. (2006). Prefazione: Avicenna e la storia della metafisica. En: Avicenna. Metafisica: La scienza delle Cose Divine (pp. v- Xxxvi). (Texto árabe, texto latino, traducción italiana, notas y aparato de O. Lizzini; prefacio y edición de P. Porro). Milano: Bompiani.

Porro, P. (2014). Henry of Ghent. En: E. N. Zalta (Ed.), The Stanford Encyclopedia of Philosophy (edición de otoño del 2014). Recuperado de https://plato. stanford.edu/archives/fall2014/entries/henry-ghent/

Rosemann, P. W. (2004). Peter Lombard. Nueva York: Oxford University Press.

Salinas Leal, H. H. (2015). Configuraciones de la filosofía medieval. Universitas Philosophica, 32(64), 345-367. Dor: 10.11144/Javeriana.uph32-64.cofm

Salinas Leal, H. H. (2016). Dignitas et nobilitas metaphysicae: Objeto y articulación de la metafísica en el prólogo del comentario de Duns Escoto a la Metafísica de Aristóteles. Revista Española de Filosofía Medieval, 23, 241-258. DOI: $10.21071 /$ refime.v23i.8981

Salinas Leal, H. H. (2019). El universal lógico como modus concipiendi en Duns Escoto. Anales del Seminario de Historia de la Filosofia, 36(1), 29-48. DOI: 10.5209/ASHF.63361

Sondag, G. (2005). Duns Scot. París: Vrin.

Tomás de Aquino (1888). Opera omnia (Vol. IV): Pars Prima Summae Theologiae. Commissio Leonina (Ed.). Rome: Ex Typographia Polyglotta.

Williams, T. (Ed.). (2003). Cambridge Companion to Duns Scotus. Cambridge: Cambridge University Press.

Zourabichvili, F. (2003). Le vocabulaire de Deleuze. París: Ellipses. 